\title{
Percutaneous dilatational tracheostomy versus fibre optic bronchoscopy-guided percutaneous dilatational tracheostomy in critically ill patients: a randomised controlled trial
}

\author{
Guanggui Shen ${ }^{1} \cdot$ Hongzhen Yin $^{1} \cdot$ Yingya Cao ${ }^{1} \cdot$ Meijun Zhang ${ }^{1} \cdot$ Jingyi Wu ${ }^{1} \cdot$ Xiaogan Jiang ${ }^{1} \cdot$ Tao $\mathbf{Y u}^{1} \cdot$ Weihua $\mathrm{Lu}^{1}$
}

Received: 22 May 2018 / Accepted: 1 August 2018 / Published online: 11 August 2018

(C) Royal Academy of Medicine in Ireland 2018

\begin{abstract}
Background To determine whether fibre optic bronchoscopy-guided percutaneous dilatational tracheostomy (FOB-PDT) is a better option in critically ill patients, we compared the efficacy and incidence of procedure complications between PDT with and without FOB.

Methods We included 90 patients with oral intubation and mechanical ventilation who received PDT with $(n=45$, FOB-PDT group) and without ( $n=45$, PDT group) FOB. For all patients, a simplification of the Griggs technique was used in this study with a central venous catheter set and dilating forceps. Demographic data, body mass index (BMI), Sequential Organ Failure Assessment (SOFA) score, Acute Physiology and Chronic Health Evaluation II (APACHE II) score, rate of first-time success, complication rate and time of procedure were evaluated in both groups. PDT was performed guided by FOB only in the FOBPDT group.

Results The rate of major complications, including minor or major haemorrhage requiring intervention and subcutaneous emphysema in the neck or pneumothorax, was significantly higher in the PDT group than in the PDT-FOB group (40\% vs. $20 \%, P<0.05)$. Significant differences were observed between the two groups with respect to the rate of first-time success $(64.4 \%$ vs. $93.3 \%, P<0.05)$; the rate of first-time success puncture with the puncture needle in the PDT-FOB group was higher than that in the PDT group $(93.3 \%$ vs. $75.6 \%, P<0.05)$. The mean procedure duration was significantly longer in the PDT group than in the PDT-FOB group $(12.9 \pm 1.1$ vs. $9.8 \pm 1.2 \mathrm{~min}, P<0.05)$.

Conclusions PDT with FOB offers the advantages of a high rate of first-time success, a low complication rate and short-procedure duration. Thus, FOB-PDT is a better option in critically ill patients.
\end{abstract}

Keywords Bronchoscopy $\cdot$ Mechanical ventilation $\cdot$ Tracheostomy

\section{Introduction}

Critically ill patients in intensive care units who require a long-term artificial airway typically received percutaneous dilatational tracheostomy (PDT) [1-4]. However, although this method is inexpensive and easy to perform at the bedside, PDT may cause serious complications, such as bleeding, tracheoesophageal fistula and perforation of the posterior wall

Tao Yu

yutao201103@gmail.com

1 Department of Critical Care Medicine, Research Center for Functional Maintenance and Reconstruction of Viscera, Wannan Medical College First Affiliated Hospital, Yijishan Hospital, Wuhu, China of the trachea [5]. For some patients, it is difficult to locate the tracheostomy site accurately based upon anatomical landmarks. During the procedure, the surgeon cannot monitor the position of the puncture needle, the guidewire or the dilating forceps directly due to the nature of this blind method.

Fibre optic bronchoscopy (FOB) is commonly used in intensive care units for these patients [4, 6-8]. FOB can be used to clear airway secretions. Using fibre optic bronchoscopy with a light source, a surgeon can easily locate the tracheostomy site accurately by transillumination of the fibre optic bronchoscope light inside the trachea. Because the fibre optic bronchoscope has a camera, the surgeon can easily monitor the percutaneous dilatational tracheostomy procedure. Data from randomised studies on percutaneous dilatational tracheostomy with or without FOB are limited [9-12]. Therefore, the present study assesses the effectiveness of locating the 
tracheostomy site accurately, the complication rates of bleeding and insertion into a false cavity and the procedure duration.

\section{Methods}

\section{Patients}

This is a prospective and randomised study. The present study was conducted among critically ill patients who had been admitted to our intensive care unit (ICU) between January 2017 and March 2018. Our study was approved by Ethics Committee of Yijishan Hospital of Wannan Medical College (2016-TG08) and registered at www.chictr.org.cn with the project number ChiCTR1800015585. Our study was approved by the Ethics Committee before patient recruitment. In the beginning, we attempted to register at clinicaltrials.gov; it took an extended period of time and the registration was unsuccessful. The administrator of the clinicaltrials.gov informed us that we could register at www. chictr.org.cn given the registration difficulties. Chinese Clinical Trial Registry was accepted as a partner of the World Health Organisation International Clinical Trial Registry Platform (WHO ICTRP). Chinese Clinical Trial Registry allows researchers to register retrospectively (supplementary registration). Therefore, we registered at www.chictr.org.cn after the study was finished. To ensure its authenticity, it is necessary to supplement the registration by providing the original data and verify it with the administrator of the Chinese Clinical Trial Registry. Those who apply for supplementary registration must provide evidence of the study, including original data, and allow public queries through public databases. As a result, any applicant for the supplementary registration will be provided with the original data through the clinical trial database public management platform ResMan for review and publicity. All procedures performed in studies involving human participants were in accordance with the ethical standards of the institutional and/or national research committee and with the 1964 Helsinki declaration and its later amendments or comparable ethical standards. Informed consent was obtained from of all individual participants included in the study. For unconscious patients, informed consent was obtained from first-degree relatives. If informed consent was not received, those patients will be excluded from the study.

All of the included ventilated patients in this study required mechanical ventilation for more than 7 days and were difficult to wean or exhibited cerebral dysfunction (Glasgow coma score less than 7). In addition, after patient evaluation, some ventilated patients required mechanical ventilation for less than 7 days, but the clinical physicians estimated the patient to need long-term mechanical ventilation and would benefit from tracheostomy.

The exclusion criteria for this study were as follows: less than 18 years of age, platelet count less than $50,000 \mathrm{~mm}^{3}$, activated partial thromboplastin time $\geq 1.5$-fold the normal value, local infection in the neck, trachea or neck abnormalities, systolic blood pressure $<90 \mathrm{mmHg}$ despite maintenance of blood pressure by continuously infusion of dobutamine more than $8 \mu \mathrm{g} / \mathrm{kg} / \mathrm{min}$ and norepinephrine more than $0.4 \mu \mathrm{g} / \mathrm{kg} / \mathrm{min}$ and prior neck surgery. In reference to previous publications [13], if the patients' systolic blood pressure was $<90 \mathrm{mmHg}$ despite maintenance of blood pressure by more than two types of vasopressive agents, this means that those patients had severe circulatory failure and were excluded from this study for the safety reasons.

\section{Study design}

Opiates and other sedative agents were used for analgesia and for maintenance of a target Richmond Agitation Sedation Scale (RASS) score of -5 . During the procedure, all of the included patients were ventilated with an oral tracheal tube, and the ventilation model was synchronised intermittent mandatory with pressure support or volume-controlled ventilation with FiO2 (the fraction of inspired oxygen) $100 \%$. Hemodynamic status, peripheral oxygen saturation and ventilator parameters were monitored routinely. Following suitable sedation, the patient's head was extended and placed in a sniff position by the placement of a $10-\mathrm{cm}$ tall soft pillow under the patient's shoulders. We observed that if local anaesthetic (usually containing vasoconstrictor solution) was used, the transillumination of the fibre optic bronchoscope light inside the tracheal lumen could not be seen clearly on the surface of the tracheal skin (Fig. 1b); thus, local anaesthetic was not required, as opiates and other sedative agents were used for analgesia and for maintenance of a target Richmond Agitation Sedation Scale (RASS) score of -5 .

\section{Measurements and data collection}

\section{Percutaneous dilatational tracheostomy}

The tracheal tube was withdrawn from the incisors to a distance of approximately $16-18 \mathrm{~cm}$. To save money, a percutaneous tracheostomy kit was not used. A simplified Griggs technique was used for percutaneous tracheostomy in this study with a central venous catheter set and dilating forceps. A 16-gauge intravenous cannula puncture needle and the guidewire from a deep venous percutaneous kit (Zhuhai Fornia Medical Equipment Co., Ltd., Guangdong, China) (Fig. 1a) were inserted into the tracheal lumen $2.0 \mathrm{~cm}$ below the cricoid cartilage or between the second and third tracheal 
Fig. 1 Percutaneous dilatational tracheostomy guided by fibre optic bronchoscopy $\mathbf{a}$

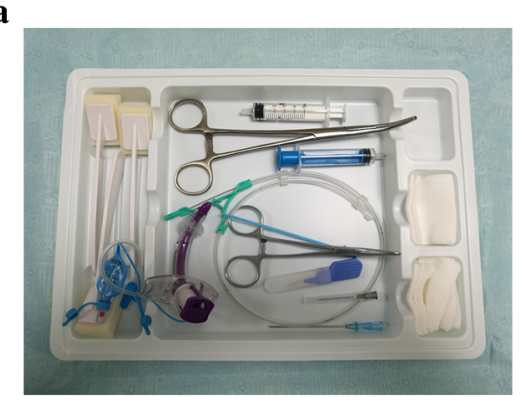

c

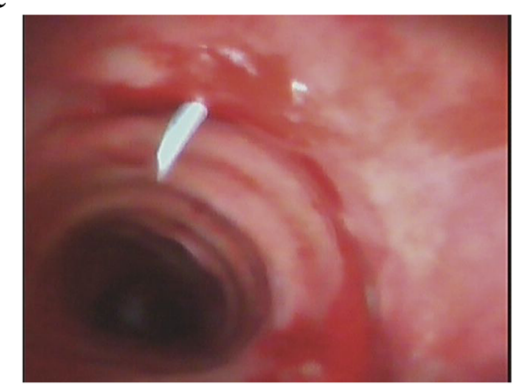

b

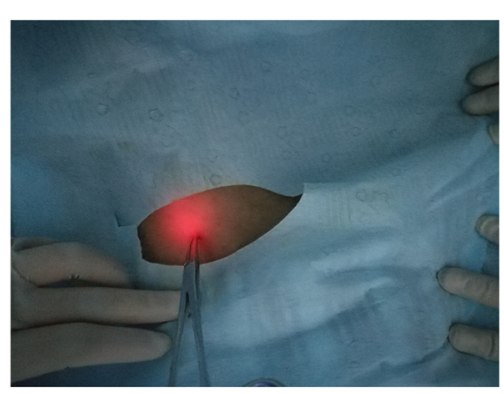

d

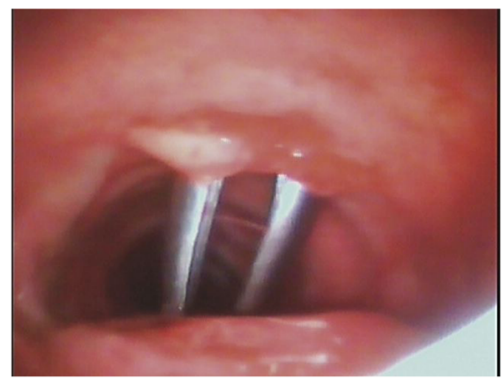

cartilage rings. The skin of the puncture site was horizontally incised to create an incision of approximately $1.5 \mathrm{~cm}$. A puncture needle with a syringe was inserted vertically into the skin until a sense of breakthrough was perceived and a large number of bubbles could be retrieved from the syringe, indicating that the puncture needle was inserted into the tracheal lumen. The guidewire was placed into the tracheal lumen $10-15 \mathrm{~cm}$ through the puncture needle. After the puncture needle was withdrawn from the tracheal lumen, the anterior cervical tissue, muscles and tracheal lumen were carefully expanded with dilating forceps (Fig. 1a). A size 7.0-8.5 tracheostomy cannula was placed into the patients' tracheal lumen guided by the guidewire after the dilating forceps were withdrawn from the tracheal lumen. The guidewire was then withdrawn from the tracheal lumen. The tracheostomy cuff was inflated, and the location of tracheostomy cannulas was confirmed according to the patients' respiratory sounds and monitoring of the respiratory wave from the mechanical ventilation. The oral endotracheal tube was then removed. After the procedure was completed, a bedside chest X-ray was obtained, and thoracic ultrasound was performed to detect the presence of pneumothorax, subcutaneous emphysema, subcutaneous bleeding and other any complications. Thoracic ultrasound was performed to detect the presence of pleural effusion, and a bedside chest $\mathrm{X}$-ray was obtained to detect the presence of pneumothorax, subcutaneous emphysema and any other complications.

\section{Percutaneous dilatational tracheostomy guided by fibre optic bronchoscopy}

A fibre optic bronchoscope was inserted through the small hole located in the cap of the oral endotracheal tube. This approach allowed the patients to be mechanically ventilated while using the fibre optic bronchoscope to monitor the percutaneous dilatational tracheostomy procedure at the same time. The tip of the fibre optic bronchoscope was positioned approximately $1.0 \mathrm{~cm}$ beyond than the tip of the oral endotracheal tube. Using the guide of the fibre optic bronchoscope, the tip of the oral endotracheal tube was withdrawn to just under the glottis until the second and third tracheal cartilage rings were visible. First, to locate the needle puncture site, the tracheal skin was gently pressed with vessel forceps. The lights of the room were turned off as necessary, and the transillumination of the fibre optic bronchoscope light inside the tracheal lumen could be seen on the surface of the tracheal skin (Fig. 1b). The tip of the puncture needle could be seen as it was inserted into the trachea lumen (Fig. 1c), and the puncture needle was adjusted and positioned in the middle of the trachea lumen. The guidewire and the dilating forceps, which were employed as described above, were visualised during the procedure using the fibre optic bronchoscope (Fig. 1d).

\section{Outcome measurements}

The first endpoints were complication rate including minor or major haemorrhage requiring intervention, pneumothorax, persistent hypotension and persistent hypoxemia. Minor haemorrhage was defined as localised stomal or intatracheal bleeding that was self-limiting or only required treatment with local compression. Major haemorrhage was defined as bleeding that could not be successfully treated with only local compression or continuous stomal or intatracheal haemorrhage requiring transfusion or emergency surgical intervention [1, 11]. Persistent hypotension was defined as systolic blood 
pressure less than $90 \mathrm{mmHg}$ for more than 5 min despite fluid infusion, use of vasopressor agents or increased doses of vasopressors agents. Persistent hypoxemia was defined as peripheral oxygen saturation less than $90 \%$ for more than 5 min during the procedure [7]. The secondary endpoints were total rate of first-time success, time of procedure, 28-day mortality, abandoned treatment and automatic discharge.

\section{Statistical analysis}

The Kolmogorov-Smirnov test was used to test the normality of distribution of the data including age, Acute Physiology and Chronic Health Evaluation II (APACHE II) score, body mass index (BMI) and Sequential Organ Failure Assessment (SOFA) score. All data were expressed as the mean \pm standard deviation $(\mathrm{M} \pm \mathrm{SD})$. We compared the data between the two groups of patients using an independent $t$ test, Pearson $\chi 2$ and Fisher exact test applied as appropriate. A $P<0.05$ was considered to be statistically significant. SPSS 16.0 software package (SPSS Inc., Chicago, IL, USA) was used to conduct statistical analyses.
The present study used a sample size of 45 subjects in both the PDT-FOB and PDT groups based on previous studies [1]; a total of 80 patients with 40 in each group were suitable to ensure an $\alpha$ error of 0.05 and $\beta$ error of 0.8 . However, considering the heterogeneity in the intensive care unit population, the sample size of the present study was planned to include a total of 90 patients, 45 in each group.

\section{Results}

From January 2017 to March 2018, a total of 90 critically ill patients receiving tracheostomy were randomised to either the PDT or PDT-FOB group (See Fig. 2). Patients' baseline characteristics are shown in Table 1. Among the included patients, there were 67 male patients and 23 female patients. Patients were 24-90 years old, with an average age of $62 \pm 15$ years and an average BMI of $22 \pm 3 \mathrm{~kg} / \mathrm{m}^{2}$. No significant differences were observed between the two groups with respect to age, gender, APACHEIIscore, SOFA score, time of oral intubation before tracheotomy, or 28-day mortality (with all $P>$
Fig. 2 Consolidated Standards of Reporting Trials flowchart. FOBPDT, fibre optic bronchoscopyguided percutaneous dilatational tracheostomy

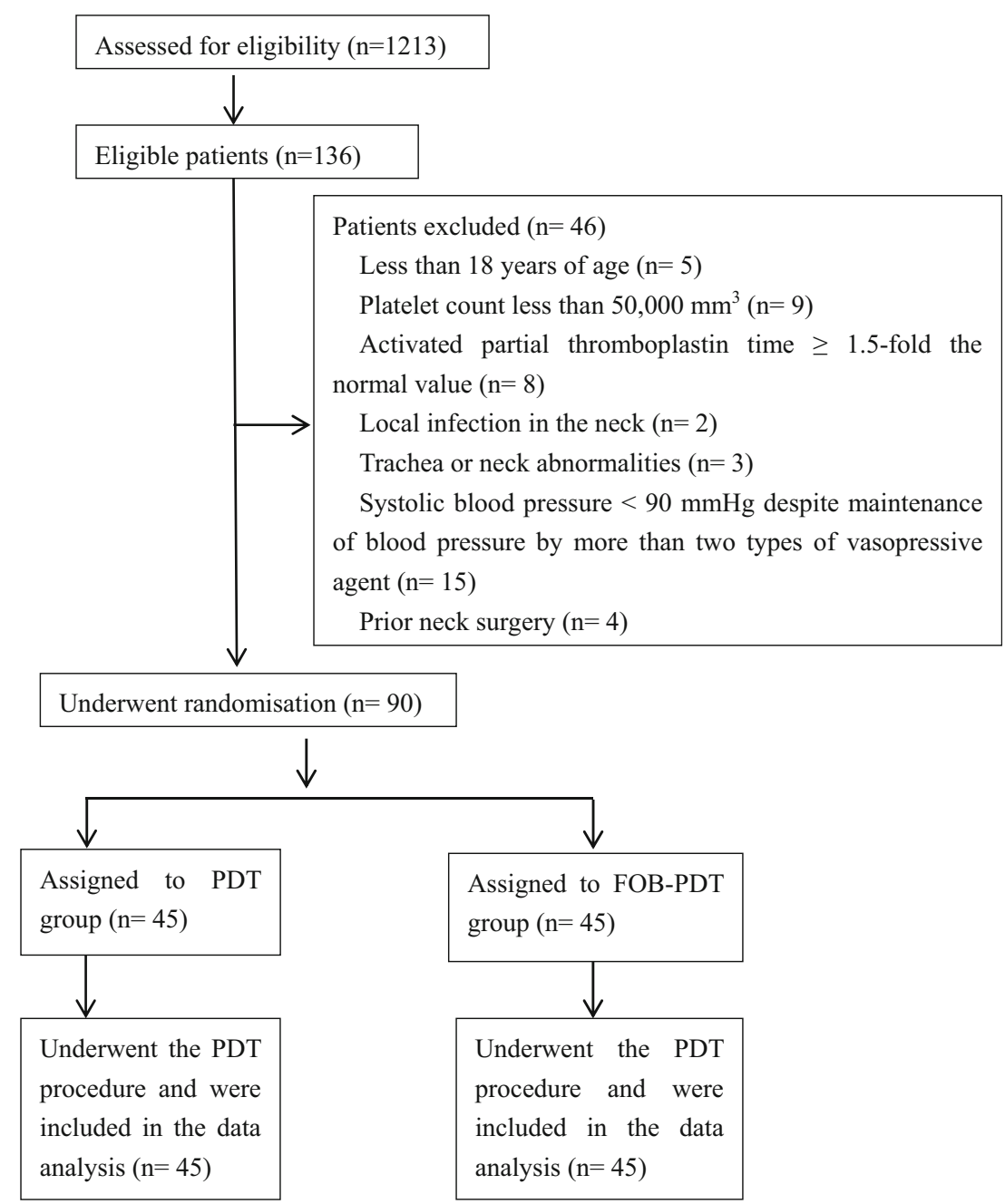


Table 1 Baseline patient characteristics

\begin{tabular}{|c|c|c|c|c|c|}
\hline Characteristic & All $(n=90)$ & PDT $(n=45)$ & PDT-FOB $(n=45)$ & $t / \chi^{2}$ & $P$ \\
\hline Age (years) & $62 \pm 15$ & $63 \pm 16$ & $61 \pm 14$ & -0.670 & 0.504 \\
\hline Gender (female/male), $n$ & $23 / 67$ & $13 / 32$ & $10 / 35$ & 0.305 & 0.581 \\
\hline Body mass index $\left(\mathrm{kg} / \mathrm{m}^{2}\right)$ & $22 \pm 3$ & $23 \pm 3$ & $22 \pm 3$ & 0.352 & 0.726 \\
\hline APACHEIIscore & $23 \pm 7$ & $22 \pm 7$ & $24 \pm 7$ & -0.902 & 0.369 \\
\hline SOFA score & $8 \pm 3$ & $9 \pm 3$ & $8 \pm 4$ & 1.256 & 0.213 \\
\hline 28-day mortality $(\%)(n)$ & $20.0 \%(18 / 90)$ & $22.2 \%(10 / 45)$ & $17.7 \%(8 / 45)$ & 0.278 & 0.598 \\
\hline Abandoned treatment and/or automatic discharge $(\%)(n)$ & $22.2 \%(20 / 90)$ & $24.4 \%(11 / 45)$ & $20.0 \%(9 / 45)$ & 0.257 & 0.800 \\
\hline Duration of oral intubation before tracheotomy, days & $7 \pm 4$ & $7 \pm 4$ & $7 \pm 4$ & 0.146 & 0.884 \\
\hline Number of patients receiving anti-platelet medications $(\%)(n)$ & $21.1 \%(19 / 90)$ & $22.2 \%(10 / 45)$ & $20.0 \%(9 / 45)$ & 0.067 & 0.796 \\
\hline Requirement for platelet or blood transfusion $(\%)(n)$ & $14.4 \%(13 / 90)$ & $13.3 \%(6 / 45)$ & $15.6 \%(7 / 45)$ & 0.090 & 0.764 \\
\hline Continuous dialysis $(\%)(n)$ & $21.1 \%(19 / 90)$ & $24.4 \%(11 / 45)$ & $17.8 \%(8 / 45)$ & 0.600 & 0.438 \\
\hline \multicolumn{6}{|l|}{ Indication for percutaneous dilatational tracheostomy } \\
\hline Respiratory failure $(\%)(n)$ & $68.9 \%(62 / 90)$ & $71.1 \%(32 / 45)$ & $66.7 \%(30 / 45)$ & 0.207 & 0.649 \\
\hline Poor neurological status $(\%)(n)$ & $31.1 \%(28 / 90)$ & $28.3 \%(13 / 45)$ & $33.3 \%(15 / 45)$ & 0.207 & 0.649 \\
\hline
\end{tabular}

0.05). During the procedure, all patients' peripheral oxygen saturation was kept at more than $96 \%$; no hypertension or serious arrhythmia was observed in either group.

The complication rate, including minor or major haemorrhage requiring interventions and subcutaneous emphysema in the neck or pneumothorax, in the PDT group was significantly higher than that in the PDT-FOB group ( $40 \%$ vs. $20 \%$, $P<0.05$ ) (Table 2). Minor haemorrhage controlled by compression was observed in $15(33.3 \%)$ patients in the PDT group and in $9(20 \%)$ patients in the PDT-FOB group. Unfortunately, two cases of major haemorrhage requiring intervention were observed in the PDT group, and the patients were immediately transferred to the operation room, where they received surgical haemostasis by an otolaryngologist. One case of subcutaneous emphysema in the neck and pneumothorax was observed in the PDT group, and the patient received closed drainage of the thoracic cavity. No major haemorrhage requiring intervention, subcutaneous emphysema or pneumothoraxes were observed in the PDT-FOB group.

Significant differences were observed between the two groups with respect to the rate of first-time success $(64.4 \%$ vs. $93.3 \%, P<0.05)$. The rate of first-time success puncture with the puncture needle in the PDT-FOB group was higher than that in the PDT group (3.3\% vs. $75.6 \%, P<0.05)$; in the PDT-FOB group, the rate of first-time success with insertion of the tracheostomy catheter was $100 \%$. Tracheostomy catheter insertion attempts were performed in five patients (rate of firsttime success for insertion of the tracheostomy catheter was $88.9 \%$ ) in the PDT group (Table 2). The mean procedure duration was significantly longer in the PDT group than the PDTFOB group (12.9 \pm 1.1 vs. $9.8 \pm 1.2 \mathrm{~min}, P<0.05$; Table 2$)$.

\section{Discussion}

Although similar haemorrhage and subcutaneous emphysema rates were seen between PDT and PDT-FOB, there were more cases of total complications in the PDT group. Additionally, there was one case of major haemorrhage in the PDT-FOB group and one case with pneumothorax in the PDT-FOB group. This serious complication may be a consequence of the blind nature of the method in the PDT group. We noticed

Table 2 Incidence of complications

\begin{tabular}{|c|c|c|c|c|c|}
\hline Characteristic & All $(n=90)$ & $\operatorname{PDT}(n=45)$ & PDT-FOB $(n=45)$ & $t / \chi^{2}$ & $P$ \\
\hline Complication rate $(\%)(n)$ & $30 \%(27 / 90)$ & $40 \%(18 / 45)$ & $20 \%(9 / 45)$ & 4.286 & 0.038 \\
\hline Minor haemorrhage controlled by compression $(\%)(n)$ & $26.7 \%(24 / 90)$ & $33.3 \%(15 / 45)$ & $20 \%(9 / 45)$ & 2.045 & 0.153 \\
\hline Major haemorrhage requiring intervention $(\%)(n)$ & $1.1 \%(1 / 90)$ & $4.4 \%(2 / 45)$ & $0(0 / 45)$ & - & 0.494 \\
\hline Pneumothorax $(\%)(n)$ & $1.1 \%(1 / 90)$ & $2.2 \%(1 / 45)$ & $0(0 / 45)$ & - & 1.000 \\
\hline Total rate of first-time success $(\%)(n)$ & $78.9 \%(71 / 90)$ & $64.4 \%(29 / 45)$ & $93.3 \%(42 / 45)$ & 11.27 & 0.001 \\
\hline Puncture with the puncture needle $(\%)(n)$ & $84.4 \%(76 / 90)$ & $75.6 \%(34 / 45)$ & $93.3 \%(42 / 45)$ & 5.414 & 0.039 \\
\hline Insertion of the tracheostomy catheter $(\%)(n)$ & $94.4 \%(85 / 90)$ & $88.9 \%(40 / 45)$ & $100 \%(45 / 45)$ & 5.294 & 0.056 \\
\hline Time of procedure (min) & $11.3 \pm 1.3$ & $12.9 \pm 1.1$ & $9.8 \pm 1.2$ & 12.812 & $<0.001$ \\
\hline
\end{tabular}


that under direct vision of the fibre optic bronchoscope, although there is no bleeding on the surface of the skin, bleeding from the puncture point will flow into the trachea along the inner tube of the trachea. With an increase in the number of needle puncture attempts, this puncture point bleeding is more obvious. Blood secretions from the airway also increase. Repeated puncture attempts with the puncture needle or inappropriate dilation with forceps may injure the vasculature or tissue around the puncture sites. FOB allows the location of the tracheostomy site accurately by transillumination of the fibre optic bronchoscope light inside the trachea. The surgeon did not require repeat puncture attempts with the puncture needle, which may have reduced the number of cases of total complications in the PDT-FOB group. Furthermore, the low complication rate in the PDT-FOB group may be attributed to the FOB providing valuable information about the position of the tip of the dilating forceps such that the tracheal lumen and the tissue around the trachea did not need to be dilated excessively. Most importantly, using the FOB as a guide, the tracheostomy catheter would not be inserted into a false cavity.

Our data indicate that PDT-FOB had a short procedure duration. FOB allowed the surgeons to monitor the entire PDT procedure, which had a shorter duration for several reasons. First, FOB increased the ease of locating the tracheostomy site accurately by transillumination of the fibre optic bronchoscope light inside the trachea. Gently pressing the tracheal skin using a vessel forceps and turning off the lights in the room as needed allowed the transillumination of the fibre optic bronchoscope light inside the trachea lumen to successfully reveal the site of tracheal incision, especially in patients with short and wide necks. In our study, the rate of first-time success of puncture with the puncture needle in the PDT group guided by FOB was significantly higher than that in the PDT group. The surgeon did not need to adjust the puncture needle multiple times to find the correct position. Second, the aspiration of bronchial secretions at any time in the PDT guided by FOB group helped the procedure. Third, by monitoring the fibre optic bronchoscope directly, the tip of the puncture needle, the guidewire and the dilating forceps were seen during the procedure. Thus, in this group, the surgeon did not need to spend more time making sure that the tip of the puncture needle, the guidewire and the dilating forceps were inserted into the trachea lumen at a correct position. End-tidal $\mathrm{CO}_{2}$ monitoring was not used in any patient during PDT in this study; the position of the tracheal tube was confirmed by respiratory measurements including tidal volume, peak airway pressure and flow curve.

Gobatto et al. reported an $8.6 \%$ rate of minor bleeding in the patients with bronchoscopy-guided percutaneous dilational tracheostomy [7]. Grensemann et al. reported an $8.7 \%(2 / 23)$ rate of adverse events including tube dislodgement during the intervention and pneumothorax after the intervention in the patients with bronchoscopy-guided percutaneous dilatational tracheostomy [6]. Kost et al. reported a
$9.2 \%$ total complication rate in the patients with endoscopic percutaneous dilatational tracheostomy [14]. In the present study, a $20 \%$ complication rate was observed in the patients in the PDT-FOB group. The probable reason may be that, in order to reduce hospitalisation costs, we used a simplification of the Griggs technique in this study with a central venous catheter set and dilating forceps. This technique, which is not commonly performed in Europe, has largely been superseded by the "single-step dilation" tracheostomy [4]. Another probable reason may be that a 16-gauge intravenous cannula puncture needle from a central venous catheter set is larger than the 14-gauge needle from a tracheostomy kit [14]. Thus, our modification may impact and potentially increase the rate of complications. Vargas et al. showed that performing PDT solely by landmark technique without any guiding technique is uncommon today [4], It is likely that their survey lacked data from China, because to my knowledge, performing PDT solely by landmark technique without any guiding technique is still very common today in China. Dinh et al. reported a $33.3 \%$ complication rate including hypoxemia, minor bleeding, major bleeding and tracheal ring break in patients $(n=12)$ with landmark technique percutaneous dilatational tracheostomy [15]. Rudas et al. observed a $37 \%$ rate of total procedural complications in the patients with landmark technique percutaneous dilatational tracheostomy, which was higher than that of real-time ultrasound guidance [16]. In the present study, the total complication rate was $40 \%$ in the PDT group patients. Our findings are consistent with previous studies $[15,16]$.

\section{Limitations}

There were several limitations in the present study. First, we did not use ultrasound before the surgery to detect the location of the thyroid gland and vascular structures in the neck. Several studies $[11,17,18]$ demonstrated that screening of the neck with ultrasound could reduce the risk of haemorrhage and other complications. The role of ultrasound screening is at the start of the procedure. Ultrasound may assist the PDT procedure by defining relevant anatomy, identifying the tracheal midline and blood vessels adjacent to the PDT insertion site, estimating tracheal depth from the skin surface and identifying patients unsuitable for the procedure. However, at the beginning of the present study, we did not have an ultrasound machine in our unit; all the ultrasound examination work was conducted by doctors in the ultrasound department, who brought an ultrasound machine to our unit temporarily. If the ultrasound machine or other visualisation machine is available to us in the future, we will use ultrasound or another visualisation machine by ourselves to assist the PDT procedure.

Second, although the duration of the FOB-PDT procedure was shorter than that of PDT, we did not measure the preparation time of FOB or the time required for transillumination with the fibre optic bronchoscope light inside the trachea 
lumen to reveal the site of tracheal incision. Moreover, the FOB-PDT procedure is affected by the personnel; the procedure cannot be performed if the FOB machine is not available or if the operator is not present, especially during a holiday. Third, in the present study, a single-centre population was included. A multi-centre study including a larger patient population is needed. Fourth, arterial blood gases (ABGs) immediately before and after the two procedures were not measured. This is important because the presence of the FOB in the tube could increase the total resistance of the system, which may result in potential worsening of ABGs in PDT-FOB. However, Saritas et al. [1] showed that FOB could increase the ratio of partial oxygen pressure to the fraction of inspired oxygen, potentially because FOB could aspirate effectively during the procedure. In the present study, worsening of peripheral oxygen saturation, hypertension or serious arrhythmia was not observed in the patients in the FOB-PDT groups.

\section{Conclusion}

In conclusion, FOB-PDT offers the advantages of a shortprocedure duration, low complication rate and high rate of first-time success. Therefore, FOB-PDT is a safe procedure that may be recommended in intensive care units for critically ill patients.

Acknowledgments This study is financially supported by the Natural Science Foundation of Anhui University (KJ2016A730 and KJ2016A718) and the Anhui Provincial Natural Science Foundation (1608085MH199).

Authors' contributions G.S. and T.Y. designed the present study. Data analysis was conducted by H.Y. and Y.C. All the authors were involved in the execution of the present study. All the authors participated in preparation of the manuscript and approved its final version for publication.

\section{Compliance with ethical standards}

Conflict of interest The authors declare that they have no conflict of interest.

\section{References}

1. Saritas A, Kurnaz MM. (2017) Comparison of bronchoscopyguided and real-time ultrasound-guided percutaneous dilatational tracheostomy: safety, complications, and effectiveness in critically ill patients. J Intensive Care Med. https://doi.org/10.1177/ 0885066617705641

2. Delaney A, Bagshaw SM, Nalos M (2006) Percutaneous dilatational tracheostomy versus surgical tracheostomy in critically ill patients: a systematic review and meta-analysis. Crit Care 10:R55. https://doi.org/10.1186/cc4887

3. Freeman BD, Isabella K, Cobb JP et al (2001) A prospective, randomized study comparing percutaneous with surgical tracheostomy in critically ill patients. Crit Care Med 29:926-930
4. Vargas M, Sutherasan Y, Antonelli M, Brunetti I, Corcione A, Laffey JG, Putensen C, Servillo G, Pelosi P (2015) Tracheostomy procedures in the intensive care unit: an international survey. Crit Care 19:291. https://doi.org/10.1186/s13054-015-1013-7

5. Ciaglia P, Firsching R, Syniec C (1985) Elective percutaneous dilatational tracheostomy. A new simple bedside procedure; preliminary report. Chest 87:715-719

6. Grensemann J, Eichler L, Kahler S et al (2017) Bronchoscopy versus an endotracheal tube mounted camera for the periinterventional visualization of percutaneous dilatational tracheostomy - a prospective, randomized trial (VivaPDT). Crit Care 21:330. https://doi.org/10.1186/s13054-017-1901-0

7. Gobatto AL, Besen BA, Tierno PF et al (2016) Ultrasound-guided percutaneous dilational tracheostomy versus bronchoscopy-guided percutaneous dilational tracheostomy in critically ill patients (TRACHUS): a randomized noninferiority controlled trial. Intensive Care Med 42:342-351. https://doi.org/10.1007/s00134016-4218-6

8. Hinerman R, Alvarez F, Keller CA (2000) Outcome of bedside percutaneous tracheostomy with bronchoscopic guidance. Intensive Care Med 26:1850-1856

9. Ravi PR, Vijay MN (2015) Real time ultrasound-guided percutaneous tracheostomy: is it a better option than bronchoscopic guided percutaneous tracheostomy? Med J Armed Forces India 71:158164. https://doi.org/10.1016/j.jcrc.2014.09.011

10. Saritas A, Saritas PU, Kurnaz MM, Beyaz SG, Ergonenc T (2016) The role of fiberoptic bronchoscopy monitoring during percutaneous dilatational tracheostomy and its routine use into tracheotomy practice. J Pak Med Assoc 66:83-89

11. Gobatto AL, Besen BA, Tierno PF et al (2015) Comparison between ultrasound- and bronchoscopy-guided percutaneous dilational tracheostomy in critically ill patients: a retrospective cohort study. J Crit Care 30:213-220. https://doi.org/10.1016/j.jcrc. 2014.09.011

12. Chacko J, Gagan B, Kumar U, Mundlapudi B (2015) Real-time ultrasound guided percutaneous dilatational tracheostomy with and without bronchoscopic control: an observational study. Minerva Anestesiol 81:166-174

13. Riker RR, Shehabi Y, Bokesch PM, Ceraso D, Wisemandle W, Koura F, Whitten P, Margolis BD, Byrne DW, Ely EW, Rocha MG, SEDCOM (Safety and Efficacy of Dexmedetomidine Compared With Midazolam) Study Group (2009) Dexmedetomidine vs midazolam for sedation of critically ill patients: a randomized trial. JAMA 301:489-499. https://doi.org/10. 1001/jama.2009.56

14. Kost KM (2005) Endoscopic percutaneous dilatational tracheotomy: a prospective evaluation of 500 consecutive cases. Laryngoscope 115:1-30. https://doi.org/10.1097/01.MLG. 0000163744.89688.E8

15. Dinh VA, Farshidpanah S, Lu S, Stokes P, Chrissian A, Shah H, Giri P, Hecht D, Nguyen HB (2014) Real-time sonographically guided percutaneous dilatational tracheostomy using a long-axis approach compared to the landmark technique. J Ultrasound Med 33:14071415. https://doi.org/10.7863/ultra.33.8.1407

16. Rudas M, Seppelt I, Herkes R, Hislop R, Rajbhandari D, Weisbrodt L (2014) Traditional landmark versus ultrasound guided tracheal puncture during percutaneous dilatational tracheostomy in adult intensive care patients: a randomised controlled trial. Crit Care 18: 514. https://doi.org/10.1186/s13054-014-0514-0

17. Rajajee V, Fletcher JJ, Rochlen LR, Jacobs TL (2011) Real-time ultrasound-guided percutaneous dilatational tracheostomy: a feasibility study. Crit Care 15:R67. https://doi.org/10.1186/cc10047

18. Sustic A, Zupan Z, Antoncic I (2004) Ultrasound-guided percutaneous dilatational tracheostomy with laryngeal mask airway control in a morbidly obese patient. J Clin Anesth 16:121-123. https://doi. org/10.1016/j.jclinane.2003.04.005 